\section{Novel approaches to the diagnosis and treatment of breast cancer}

Ismail Jatoi ${ }^{*, 1} \&$ John RBenson ${ }^{2}$ 36th San Antonio Breast Cancer Symposium, San Antonio, TX, USA, 10-14 December 2013

Progress in the treatment of breast cancer continues to require the thoughtful planning of new clinical trials and patient participation in those trials. During the 2013 San Antonio Breast Cancer Symposium (SABCS), the results of several clinical trials with practice-changing implications were presented. Additionally, the results of numerous interesting and provocative observational studies were reported. There were also several plenary lectures during the 2013 SABCS that addressed timely and controversial topics. The SABCS is a global forum for clinicians and scientists who are committed to eradicating the burden of breast cancer mortality worldwide. In this report, we highlight only a few of the many important studies and plenary talks presented during the 2013 SABCS.

The 36th annual San Antonio Breast Cancer Symposium (SABCS) was held on 10-14 December 2013, in San Antonio (TX, USA). Approximately 7700 clinicians and scientists from around the world participated in the symposium, with a wide range of presentations and keynote talks pertaining to breast cancer screening, prevention and locoregional and systemic therapies. In this report, we highlight just a few of the many important topics and studies discussed.

During the first day of the symposium, there was a educational session that focused on the global trends in breast cancer incidence and mortality. Peggy Porter of the University of Washington (WA, USA) pointed out that approximately 1.6 million new cases of breast cancer are diagnosed annually worldwide, with approximately $45 \%$ of these cases and $55 \%$ of deaths from breast cancer occurring in low-/middle-income countries. More developed countries have a typical mortality/incidence ratio of 0.27 compared with 0.44 for emerging nations. Breast cancer incidence is increasing, particularly in parts of the world where the burden of this disease was historically low. For example, since 1973, breast cancer incidence has doubled in Japan, Singapore and Korea, and increased by approximately $20-30 \%$ in Columbia and the conurbations of China and India. Developing countries have a higher proportion of premenopausal breast cancer, which are more likely to be estrogen receptor (ER) negative and, as pointed out by William Anderson of the National Cancer Institute in the USA, trends in breast cancer incidence vary according to tumor subtype. Thus, US rates of ER-negative disease are decreasing ( $0.91 \%$ per year), while the rates of ER-positive

'Division of Surgical Oncology, University of Texas Health Science Center, San Antonio, TX, USA

${ }^{2}$ Cambridge Breast Unit, Addenbrooke's Hospital, Cambridge, UK

*Author for correspondence: jatoi@uthscsa.edu

\section{KEYWORDS}

- breast cancer

- chemoprevention • local therapy • systemic therapy - overdiagnosis 
disease have been slowly rising (1.14\% per year), for reasons that are not entirely clear. Donald Berry of the MD Anderson Cancer Center (TX, USA) noted that breast cancer mortality rates have declined by approximately $25-36 \%$ in the industrialized world since approximately 1990 and approximately $1 \%$ per year since 2000 in the USA. Most of the decline is evident in white nonhispanic women and seems mostly owing to better treatments, although mammography screening is probably contributory as well. Berry anticipates that further declines in breast cancer mortality will be driven almost entirely by better treatments.

Worldwide, anywhere from 5 to $20 \%$ of all breast cancer patients present with clinical evidence of distant metastases at the time of initial diagnosis. There has been considerable controversy regarding the role of local therapy in these patients, although most clinicians have generally opted for systemic therapy alone. At the 2013 SABCS, two trials were presented that addressed this controversy. Rajendra Badwe and his colleagues conducted a prospective randomized trial at the Tata Memorial Hospital in Mumbai (India), to which they recruited 350 women with metastatic breast cancer who had an objective tumor response to six cycles of chemotherapy. These women were then randomized to receive either locoregional therapy (LRT) or not. Patients in the LRT arm underwent partial or complete surgical removal of the involved breast and axillary lymph node dissection followed by radiotherapy, while those in the no-LRT am received no surgery or radiotherapy. After 2 years of follow-up, there was no difference in overall survival (OS) between patients in the two arms of the trial, suggesting that, in the metastatic setting, local therapy has no benefit on mortality.

Similarly, a trial in Turkey (protocol MF0701) under the direction of Atilla Soran (University of Pittsburgh Medical Center, PA, USA), randomized 278 women who presented with metastatic breast cancer to receive either LRT or not soon after initial diagnosis. LRT consisted of either mastectomy or breast conserving surgery with level one and level two axillary clearance in clinically or sentinel lymph node positive patients. Radiotherapy to the whole breast was administered to patients who underwent breast conserving surgery. Standard systemic therapy was administered to all patients either immediately after randomization (the no-LRT group), or after surgical resection of the intact primary tumor (the LRT group). At 54 months, there was no significant difference in OS between patients in the LRT group versus no-LRT group. These results were entirely consistent with those reported from the Indian trial.

H Gilbert Welch of Dartmouth University (NH, USA) presented a plenary lecture on the topic of screening mammography and overdiagnosis. Mammography screening is a major public health intervention, widely utilized in many westernized countries, with mortality reductions of approximately $20 \%$. It has been associated with overdiagnosis. This refers to the detection of in situ and invasive cancers that might never have become clinically relevant in the absence of screening. Although mammography screening reduces breast cancer mortality, it is important that women consider overdiagnosis and other risks such as false positives and psychological duress, and make informed decisions about whether or not to undergo screening. The extent of overdiagnosis has been extensively debated in recent years, and will likely remain an important public issue in the years ahead. With estimated rates of $20-25 \%$, one in four screen-detected cancers pose no health risk and the adage 'better when found but perhaps better not to be found' has relevance!

Since a NIH consensus conference in 1991, breast conserving therapy (BCT) has been widely viewed as the preferred standard for local therapy of early-stage breast cancer. BCT refers to lumpectomy combined with radiotherapy and whole breast irradiation. However, there remains much uncertainty as to what constitutes an adequate surgical margin following lumpectomy. Worldwide, six randomized trials have compared lumpectomy with radiotherapy versus mastectomy for the treatment of primary breast cancer, with considerable heterogeneity amongst the trials with respect to definition of a negative margin and width of surgical margin clearance following lumpectomy. In the USA and Europe, approximately $20 \%$ of women undergo re-operation following lumpectomy for invasive breast cancer to obtain a 'satisfactory clear margin'. Rates of re-excision for lesions with a component of ductal carcinoma in situ may be as high as 30\% and the margin mandate for invasive and noninvasive disease is likely to be different. Monica Morrow of the Memorial Sloan-Kettering Cancer Center (NY, USA) addressed this topic in her plenary lecture. Her group examined rates of local 
recurrence for patients with close $(<2 \mathrm{~mm})$ or clear $(\geq 2 \mathrm{~mm})$ margins after BCT and found no statistically significant difference in local recurrence nor OS for these two margin categories. She argued that "no tumor on ink" constitutes a clear margin for invasive disease (this was the requirement in the NSABP-06 lumpectomy vs mastectomy trial). A negative margin does not imply absence of residual disease within the remaining breast tissue, but suggests that any residual tumor burden is sufficiently low to be controlled with adjuvant treatments (radiotherapy and chemo-I hormonal therapies). Morrow argued that adherence to this guideline will dramatically reduce reoperation rates following lumpectomy, and will not adversely affect the risk of local recurrences following BCT. A poster presentation by one of the authors (John R Benson, Addenbrookes Hospital, Cambridge, UK) confirmed that more than $80 \%$ of re-excisions were carried out for 'positive' margins of $<2 \mathrm{~mm}$ with no significant change in rates of re-excision for a change in minimal margin width from 5 to $2 \mathrm{~mm}$. Local recurrence is determined by a combination of surgery, tumor biology, radiation and systemic therapies.

There has been much debate concerning the role of adjuvant bisphosphonate therapy in primary breast cancer, with 15 years worth of data yielding inconsistent results and no clear message. Robert Coleman and his colleagues from the University of Sheffield (UK), conducted a meta-analysis, incorporating 36 trials of adjuvant bisphosphonate therapy, with a total of 17,791 pre- and post-menopausal women in these trials. The authors concluded that, in postmenopausal women, adjuvant bisphosphonate therapy reduces the risk of bone metastases by $34 \%$ and breast cancer mortality by $17 \%$, while no benefit is seen for premenopausal women. In postmenopausal women, adjuvant bisphosphonates reduced the risk of first distant recurrences in bone only, with no statistically significant effect at sites other than bone. The benefit of adjuvant bisphosphonate therapy in postmenopausal women was not affected by ER status, node status and use versus nonuse of chemotherapy. Furthermore, there was no difference in benefit according to type of agent used (aminobisphosphonate or clodronate) and it was commented that bisphosphonates are relatively inexpensive drugs.

For the prevention of primary breast cancer, previous trials have shown that tamoxifen, raloxifene and exemestane are effective. Results of the IBIS II trial were presented by Jack Cuzick of Queen Mary College, University of London (UK). This trial randomized almost 4000 highrisk postmenopausal women to receive either 5 years of anastrozole $(n=1944)$ or placebo $(n=1920)$. At a median follow-up of 5 years, the study found that anastrozole reduced the risk of developing breast cancer by half $(5.6 \%$ in the placebo group and $2.8 \%$ in the anastrozole group), with 40 deaths in the anastrozole group compared with 85 in the placebo group. However, it seems unlikely that anastrozole as a preventative agent would have any effect on breast cancer mortality, as most of the cancers prevented were probably very curable. Of note, only $10 \%$ of the increase in musculoskeletal side effects in the anastrozole group was attributable to aromatase inhibitor therapy and there was a small nonsignificant increase in fracture rate (8.5 vs $7.7 \%)$.

SABCS 2013 featured several presentations on the role of tumor infiltrating lymphocytes (TiLs) as prognostic factors and the potential for immune-targeted therapy in breast cancer. In particular, Sherene Loi from the Institut Jules Bordet (Brussels, Belgium) and other groups have reported an association between the level of TiLs and pathological complete response rates in neoadjuvant chemotherapy cases. In Loi's study, for each $10 \%$ increment in TiLs, there was a corresponding increase in pathological complete response of approximately $16 \%$. TiLs are notably predictive of response to neoadjuvant chemotherapy in triple negative breast cancer (ER negative, PgR negative and HER2 negative).

Thomas Julian from the National Surgical Adjuvant Breast and Bowel Project (PA, USA) gave an update of OS at 10 years in the NSABP B-32 study according to the presence or absence of occult sentinel lymph node metastases. At 8 years, there was a slight survival advantage $(1.2 \%)$ for those without immunohistochemically detected micrometastases in the sentinel lymph nodes, but at 10 years there is a nonstatistically significant difference of $3.1 \%$, with no differences in disease-free survival (absolute difference $4.7 \%$ ) or locoregional control. The downward trend for isolated tumor cells and micrometastases was not considered clinically significant and routine immunohistochemistry cannot be justified for sentinel lymph node examination. Furthermore, at 10 years there were no differences in OS, disease-free survival or loco-regional control between sentinel lymph node biopsy alone versus combined with axillary lymph node dissection. 
The future of anti-angiogenic agents in breast cancer remains an open question following negative results of the ROSE/TRIO-12, a randomized placebo controlled trial evaluating the addition of the VEGF ramucirumab to first-line docetaxol for metastatic disease. There was no improvement in either progression-free survival nor OS, but the study author (John R Mackey, University of Alberta, Canada) is optimistic that clinical gains can be achieved with refinements of regimens and patient selection. Nonetheless, current research is perhaps moving away from anti-angiogenic and anti-growth factor strategies. Other agents under investigation, but with unclear clinical utility at the present time, include mTOR inhibitors and various tyrosine kinase inhibitors, such as dasatinib. These are being combined with aromatase inhibitors in a dual agent approach, but it appears that blocking one pathway can sometimes lead to stimulation of another with a net increase in cell proliferation.
The SABCS 2013 featured the work of numerous investigators who have worked diligently to reduce the burden of breast cancer mortality. More importantly, the symposium highlighted the courage of thousands of women from around the world who have participated in clinical trials, and ultimately made advances in the treatment of breast cancer a reality. Further progress in the treatment of breast cancer will require large clinical trials, and the willingness of women to participate in those trials.

Financial \& competing interests disclosure

The authors have no relevant affliations or financial involvement with any organization or entity with a financial interest in or financial conflict with the subject matter or materials discussed in the manuscript. This includes employment, consultancies, honoraria, stock ownership or options, expert testimony, grants or patents received or pending, or royalties.

No writing assistance was utilized in the production of this manuscript. 